\title{
Acute alloxan renal toxicity in the rat initially causes degeneration of thick ascending limbs of Henle
}

\author{
Yui Terayama ${ }^{1}$, Yasushi Kodama ${ }^{2}$, Tetsuro Matsuura ${ }^{1}$, and Kiyokazu Ozaki ${ }^{{ }^{*}}$ \\ ${ }^{1}$ Laboratory of Pathology, Faculty of Pharmaceutical Sciences, Setsunan University, 45-1 Nagaotoge-cho, Hirakata, Osaka 573-0101, \\ Japan \\ ${ }^{2}$ Laboratory of Clinicopathological Therapeutics, Faculty of Pharmaceutical Sciences, Hiroshima International University, 5-1-1 \\ Hirokoshingai, Kure, Hiroshima 737-0112, Japan
}

\begin{abstract}
Alloxan (AL) is a material well-known to induce diabetes. Prior to inducing a prolonged diabetic state, AL causes acute tubulointerstitial nephritis. However, the precise primary target site and mechanism of its nephrotoxicity remain unclear. The objective of this study was to evaluate the morphological characteristics relevant to acute renal toxicity following AL administration. Rats were intravenously treated with AL. Eight hours after AL treatment, aquaporin 1-negative and $\mathrm{Na} / \mathrm{K}$ pump-positive thick ascending limbs of Henle (TAL) were degenerated in the outer medulla. These tubular lesions progressed from the outer medulla to the cortex. At day 2 after AL treatment, the lesions reached a peak, then both proximal and distal tubules also showed degeneration and necrosis, and tubular regeneration was seen in TAL. Immunohistochemically, damaged tubular epithelium included slightly enlarged prohibitin-positive granules, but it expressed no GLUT2, which is an AL transporter. Ultrastructurally, cytoplasmic and mitochondrial swelling was detected in degenerated cells of TAL. These findings suggest that AL initially causes degeneration of TAL, and induces mitochondrial and cellular damage in the tubular epithelium without involving GLUT2. (DOI: 10.1293/tox.2016-0035; J Toxicol Pathol 2017; 30: 7-13)
\end{abstract}

Key words: alloxan, acute renal toxicity, tubular necrosis, rat

\section{Introduction}

Alloxan (AL) is a material well-known to induce diabetes in experimental animals. Glycogen accumulation in distal renal tubules, so-called Ebstein-Armanni lesions, reportedly appears in the kidney of AL-induced diabetic rats and mice with prolonged hyperglycemia. Prior to inducing a prolonged diabetic state, $\mathrm{AL}$ induces acute tubulointerstitial nephritis, consequently resulting in nephrotoxicity ${ }^{1-4}$. Renal changes, varying from swelling to necrosis, occur predominantly in the epithelia of the proximal convoluted tubules $(\mathrm{PCT})^{1,2,5}$. However, there is no report about the early stage of acute renal injury in this model. The precise primary target site of its nephrotoxicity remains unclear. Identification of nephron segments is essential to evaluate drug toxicity, and the immunohistochemical marker of tubular segments could be used for this purpose ${ }^{6}$.

$\mathrm{AL}$ is a small molecule that resembles glucose, binds the

Received: 4 May 2016, Accepted: 9 August 2016

Published online in J-STAGE: 5 September 2016

*Corresponding author: K Ozaki

(e-mail: ozaki@pharm.setsunan.ac.jp)

(C)2017 The Japanese Society of Toxicologic Pathology

This is an open-access article distributed under the terms of the Creative Commons Attribution Non-Commercial No Derivatives (by-ncnd) License $<$ http://creativecommons.org/licenses/by-nc-nd/4.0/>.
GLUT2 glucose transporter, can enter cells via the GLUT2 glucose transporter in pancreatic beta cells, generates superoxide and hydroxyl radicals, and rapidly induces a necrotic cell death in beta cells ${ }^{7}$. The earliest beta cell changes are localized to the mitochondria which shows swelling and disruption of inner and occasionally outer membranes ${ }^{8}$. In addition, AL can inhibit mitochondrial aconitase ${ }^{9}$. However, there is no report regarding GLUT2 glucose transporter and ultrastructural changes at the early stage of acute AL nephrotoxicity. The objective of this study was to evaluate the morphological characteristics relevant to the acute renal lesions due to AL toxicity.

\section{Materials and Methods}

Six-week-old male Wistar/Crlj rats were purchased from Charles River Laboratories Japan, Inc. The animals were housed in stainless steel cages at a temperature of 20 to $26^{\circ} \mathrm{C}$ and a relative humidity of 40 to $70 \%$ under a $12 / 12$ $\mathrm{hr}$ light/dark cycle; they were ventilated with filtrated fresh air and allowed free access to tap water and to a widely used standard pelletized diet for experimental rats (MF, Oriental Yeast, Tokyo, Japan). The animals were handled according to the principles for all experimental procedures, which are indicated in the Guide for the Care and Use of Laboratory Animals prepared by our institutions (Setsunan University and Hiroshima International University) and the Japanese 
Association for Laboratory Animal Science. The study was approved by the Committees for Animal Experiments of Setsunan University and Hiroshima International University. Seven-week-old rats were randomly divided into 6 groups ( $\mathrm{n}=3$ per group). Fifteen rats were intravenously treated with $50 \mathrm{mg} / \mathrm{kg} \mathrm{AL}$, and the rats in each treated group were sacrificed at 8 hours, 24 hours, 2 days, 4 days, or 7 days after treatment for histopathologic examination. This dose level was set up as a given dose according to which a rat survives for a long period of time after developing signs of diabetes, and which induces continuous glycosuria. Three rats used as controls were sacrificed without AL treatment at 7.5 weeks old. Fresh urine samples were collected. Urinary glucose levels were measured semiquantitatively using a urine test paper (Wako Pure Chemical Industries, Osaka, Japan) at 8 hours, 24 hours, 2 days, 4 days, and 7 days after treatment. Blood glucose levels were also measured semiquantitatively by the glucose oxidase method (Glutest E, Sanwa Kagaku, Aichi, Japan) at 8 hours, 24 hours, 2 days, 4 days and 7 days after treatment. Samples of fresh urine and blood from the tail vein were collected from 1:00 to 4:00 pm.

At necropsy, blood samples were collected from the animals via the abdominal aorta under deep anesthesia with ketamine (40 mg/kg IM; Ketalar, Sankyo) and xylazine (2.0 $\mathrm{mg} / \mathrm{kg}$ IM; Seractal, Bayer). Blood samples were centrifuged at 3,000 rpm for $15 \mathrm{~min}$ and stored at $-80^{\circ} \mathrm{C}$ before use for serum measurements, including measurements of blood urea nitrogen (BUN) and creatinine made as kidney function markers using an automated chemistry analyzer. Kidneys were fixed in $10 \%$ phosphate-buffered formalin, dehydrated, and then embedded in paraffin. Sections ( $4 \mu \mathrm{m}$ thick) of tissue specimens were stained with hematoxylin and eosin, and subjected to PAS reaction. For immunohistochemical examination, the sections were deparaffinized in xylene and rehydrated through graded ethanol at room temperature. Rehydrated sections were microwaved in $10 \mathrm{mM}$ citrate buffer ( $\mathrm{pH} \mathrm{6.0)}$ ) for $10 \mathrm{~min}$ at $98^{\circ} \mathrm{C}$ to retrieve the antigen. Solutions and washes were prepared between the various steps using $0.05 \mathrm{M}$ Tris buffer saline (TBS, pH 7.6) with $0.01 \%$ Tween 20 . Nonspecific endogenous peroxidase activity was blocked by exposure to $0.03 \%$ hydrogen peroxide in $100 \%$ methanol for $5 \mathrm{~min}$, and masking was conducted with $1 \%$ normal goat or horse serum in Tris buffer saline for 5 min at room temperature. Incubation was carried out overnight at $4^{\circ} \mathrm{C}$ with anti-aquaporin $1(\mathrm{AQP} 1)$ rabbit polyclonal antibody (diluted 1:500; \#AB2219; Millipore, USA), antisodium/potassium ATPase subunit alpha $1(\mathrm{Na} / \mathrm{K}$ pump) mouse monoclonal antibody (diluted 1:1000; \#05-369; Millipore, USA), anti-LAMP2 rabbit polyclonal antibody (diluted 1:200; L0668, Sigma-Aldrich, USA), and anti-prohibitin rabbit polyclonal antibody (diluted 1:100; Fitzgerald Industries International, Inc., Acton, MA, USA). These sections were subsequently rinsed with TBS plus Tween 20 , treated for $30 \mathrm{~min}$ at room temperature with a biotinylated secondary antibody (Vectastain Elite kit, PK6102, PK6101, Vec- tor Laboratories, USA), rinsed with TBS plus Tween 20, incubated for $30 \mathrm{~min}$ at room temperature with Vectastain Elite ABC reagent (Vectastain Elite kit, PK6102, PK6101, Vector Laboratories, USA), rinsed with TBS plus Tween 20, incubated in diaminobenzidine solution containing $0.01 \%$ hydrogen peroxide for the peroxidase coloring reaction, and counterstained with Mayer's hematoxylin.

Formalin-fixed tissue fragments of the kidney for electron microscopy were refixed with $2.5 \%$ glutaraldehyde in $0.1 \mathrm{M}$ phosphate buffer ( $\mathrm{pH} 7.4$ ). After fixation, the tissue samples were postfixed in $1 \%$ osmium tetroxide solution $(\mathrm{pH}$ 7.4) and processed into epoxy resin. Ultrathin sections were cut and stained with uranyl acetate and lead citrate and examined under an electron microscope (JEM 1200EX, JEOL, Tokyo, Japan).

\section{Results}

\section{Hyperglycemia, glucosuria and blood chemistry}

Hyperglycemia was observed from 8 hours after AL treatment, and severe glucosuria was detected from 24 hours after AL treatment (Table 1). The levels of BUN and creatinine increased 24 hours and 2 days after AL treatment, but they decreased 4 days after AL treatment, and recovered to the same levels as the non-treated group 7 days after AL treatment (Table 1).

\section{Tubular degeneration expanded from the outer me- dulla to the cortex}

Grossly, the kidneys showed no gross findings in any of the rats. Histopathologically, at 8 hours after AL treatment, mild granular degeneration was detected in thick ascending limbs of Henle (TAL) in the outer medulla (Fig. 1a, b). At 24 hours after AL treatment, tubular lesions gradually progressed from the outer medulla to the cortex. Severe degeneration and necrosis with tubular lumens containing cellular debris and granular substances were observed in TAL (Fig. 1c). Distal convoluted tubules (DCT) revealed granular and vacuolar degeneration with dilation of lumen (Fig. 1d), and mild vacuolation of proximal tubules was also detected. Two days after AL treatment, tubular degeneration and regeneration was apparent in TAL (Fig. 1e). Tubular lesions reached a peak at the cortex. Severe degeneration and necrosis were observed in DCT, and proximal convoluted tubules (PCT) exhibited vacuolar degeneration and necrosis with severe dilation of lumen (Fig. 1f). Four days after AL treatment, tubular regeneration progressed in all areas of the cortex, but tubular degeneration was still present in DCT. Seven days after AL treatment, necrosis of tubules disappeared, but tubular regeneration continued with dilation of lumen in TAL and DCT (Fig. 1g, h). These results demonstrate that AL-induced tubular degeneration expands from the outer medulla to the cortex. 
Table 1. Blood and Urine Glucose Levels, Final Body Weight and Renal Function

\begin{tabular}{|c|c|c|c|c|c|}
\hline Time after AL treatment & $\begin{array}{c}\text { Blood glucose } \\
(\mathrm{mg} / \mathrm{dL})\end{array}$ & $\begin{array}{l}\text { Urine } \\
\text { glucose }\end{array}$ & $\begin{array}{c}\text { Body weight } \\
(\mathrm{g})\end{array}$ & $\begin{array}{c}\text { BUN } \\
(\mathrm{mg} / \mathrm{dL})\end{array}$ & $\begin{array}{c}\text { Creatinine } \\
(\mathrm{mg} / \mathrm{dL})\end{array}$ \\
\hline Non-treated & NA & NA & $274.4 \pm 2.1$ & $24.7 \pm 1.75$ & $0.27 \pm 0.03$ \\
\hline 8 hours & $349.0 \pm 48.1$ & \pm & $277.8 \pm 2.2$ & $16.5 \pm 0.97$ & $0.20 \pm 0.01$ \\
\hline 24 hours & $392.7 \pm 48.6$ & ++++ & $268.0 \pm 2.9$ & $64.1 \pm 25.13$ & $0.63 \pm 0.25$ \\
\hline 2 days & $449.0 \pm 107.6$ & ++++ & $260.8 \pm 10.3$ & $71.7 \pm 36.04$ & $0.53 \pm 0.25$ \\
\hline 4 days & $543.0 \pm 57.0$ & ++++ & $266.7 \pm 5.5$ & $34.1 \pm 8.10$ & $0.28 \pm 0.09$ \\
\hline 7 days & $468.0 \pm 42.0$ & ++++ & $284.4 \pm 7.8$ & $27.6 \pm 4.24$ & $0.26 \pm 0.01$ \\
\hline
\end{tabular}

Symbols: $\pm: 50 \mathrm{mg} / \mathrm{dL},++++: 2,000 \mathrm{mg} / \mathrm{dL}$.

\section{AL initially caused degeneration of the thick ascend- ing limbs of Henle in the outer medulla}

To determine the primary lesion caused by AL treatment, tubule-specific markers were stained immunohistochemically. AQP1 was localized in cytoplasm of proximal tubules and descending limbs of Henle (DTL), and was negative in TAL in the non-treated group (Fig. 2a). At 8 and 24 hours after AL treatment, degenerated tubules corresponded to AQP1-negative TAL (Fig. 2b). At 24 hours after AL treatment, the brush border of the proximal tubule showed strong AQP1 expression compared with cytoplasm (Fig. 2b). The expression of $\mathrm{Na} / \mathrm{K}$ pump was localized on the basolateral proximal tubule but was distributed through all of the cytoplasm of TAL in the non-treated group (Fig. 2c). At 8 and 24 hours after AL treatment, degenerated tubules corresponded to $\mathrm{Na} / \mathrm{K}$ pump-positive TAL (Fig. 2d). The positivity level of the proximal tubule was decreased as compared with that in the non-treated group (Fig. 2d). These results reveal that AL initially causes degeneration of the thick ascending limbs of Henle in the outer medulla.

\section{AL induced mitochondrial and cellular damage in the tubular epithelium without involving GLUT2}

To identify injured subcellular sites in granular degeneration of TAL, organelle-specific markers and ultrastructural technique were used. Expression of prohibitin (a mitochondrial marker) in TAL was stronger than that in the proximal tubule in the non-treated group (Fig. 3a). At 8 hours after AL treatment, its positivity in TAL and the proximal tubule remain unchanged (Fig. 3b). At 24 hours after $\mathrm{AL}$ treatment, prohibitin-positive granules in TAL varied in size, and the staining intensity decreased (Fig. 3c). The amount and intensity of staining for LAMP2 (a lysosome marker) in TAL at 8 hours, 24 hours, 2 days, 4 days and 7 days after treatment were similar to those in the non-treated group.Ultrastructurally, extensive interdigitation with elongated mitochondria was observed in TAL in the non-treated group (Fig. 3d). At 8 hours after AL treatment, the lesions in TAL were characterized by multifocal rarefaction of cytoplasm, slight swelling of mitochondria, and dilation of the endoplasmic reticulm (Fig. 3e). These changes progressed till 24 hours after AL treatment (Fig. 3f). Severe swelling of cytoplasm and mitochondria and irregular basal interdigitation were observed in TAL. To clarify the relationship between damage of TAL and GLUT2, a transporter of AL, GLUT2 was stained immunohistochemically. GLUT2 was expressed at the basolateral membrane of PCT in the nontreated group (Fig. 4a), but there was no expression in the pars recta, TAL, and distal tubule (Fig. 4a, b). These results suggest that $\mathrm{AL}$ induces mitochondrial and cellular membrane damage in the tubular epithelium without involving GLUT2.

\section{Discussion}

Previous studies have shown that acute renal lesions 24 hours after AL treatment indicate swelling of the PCT and $\mathrm{DCT}^{2}$. Three to 5 days after AL treatment, necrosis and swelling of tubules became more evident predominantly in the proximal tubule, but was also observed in some distal tubules $^{2}$. However, our findings indicated that the primarily affected tubule after AL treatment was the TAL, and that tubular degeneration expanded from the outer medulla to the cortex. According to the expansion of lesions, the proximal tubule and distal tubule were affected. To accurately elucidate the primary lesion in the renal tubules, immunohistochemical staining of the renal tubular marker was necessary. AQP1 is reportedly expressed in the proximal tubule and DTL ${ }^{6,10}$. As previously reported, AQP1-positive tubules of this rat was consistent to the localization and morphology of the proximal tubule and DTL. The expression of $\mathrm{Na} / \mathrm{K}$ pump was reported to be strong in TAL, and mild in the proximal tubule ${ }^{11}$. In our rats, strong expression was detected in the entire cytoplasm in TAL, whereas the proximal tubule only showed basolateral staining. Although we could not find and test any specific marker for TAL, the use of both $\mathrm{AQP}-1$ and $\mathrm{Na} / \mathrm{K}$ pump staining made it possible to identify TAL in the outer medulla.

AL is a small molecule that resembles glucose, binds the GLUT2 glucose transporter, and can enter cells via the GLUT2 glucose transporter ${ }^{12-14}$. AL generates superoxide and hydroxyl radicals and rapidly induces a necrotic cell death in beta cells within 48 hours after injection? ${ }^{7}$ The GLUT2 glucose transporter is present in beta cells, hepatocytes, renal tubular cells, and small intestinal epithelial cells ${ }^{7}$. However, because beta cells have relatively weak defenses against oxidative stress, they are especially sensitive to free radical-mediated damage caused by $\mathrm{AL}^{7}$. The 


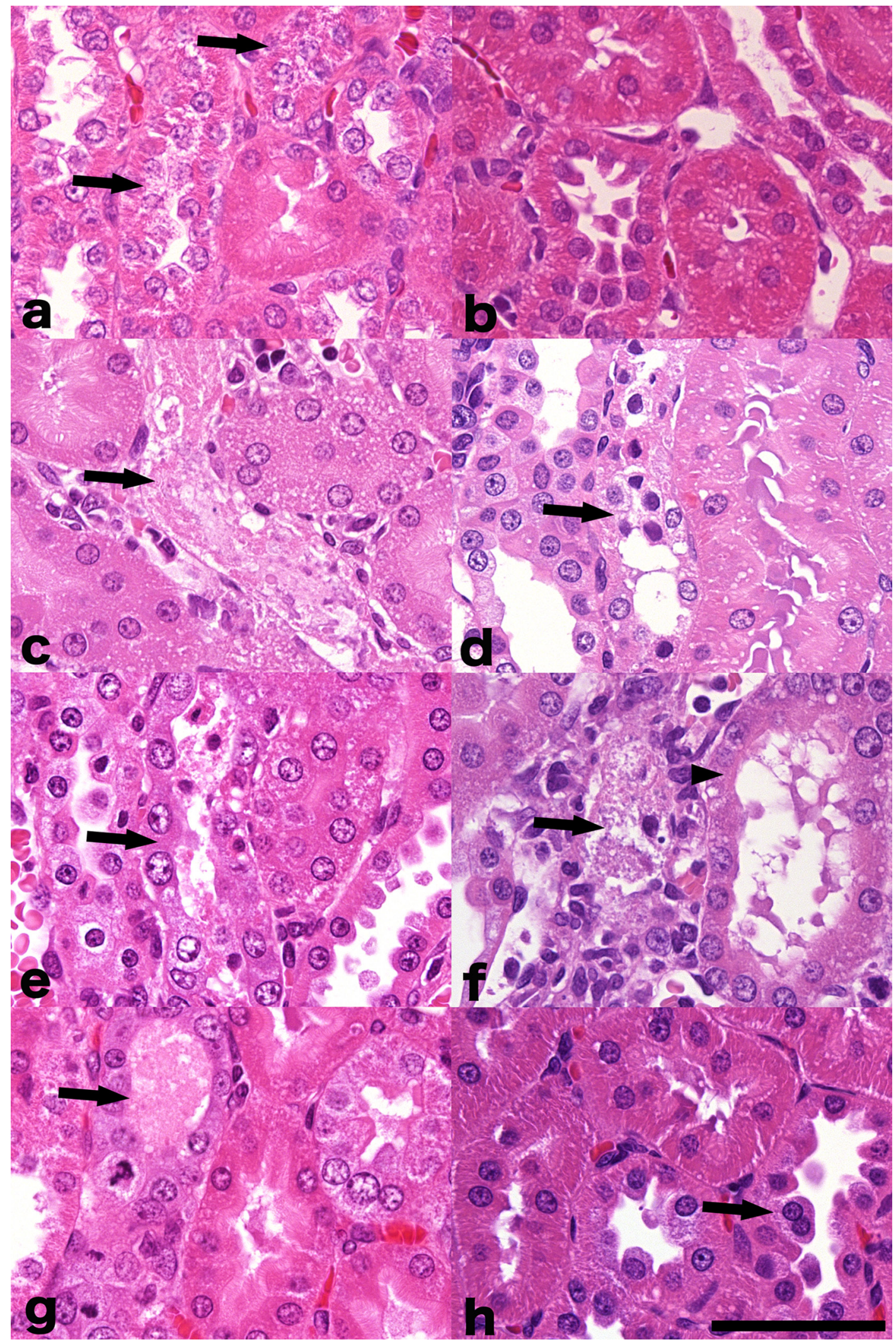

Fig. 1. Tubular degeneration expands from the outer medulla to the cortex. (a, b) At 8 hours after AL treatment, mild granular degeneration is observed in TAL (a, arrows). (c, d) At 24 hours after AL treatment, tubular necrosis is noted in TAL (c, arrow). Granular and vacuolar degeneration is observed in DCT (d, arrow). (e, f) At 2 days after AL treatment, tubular regeneration is seen in TAL (e, arrow). Degeneration and necrosis are apparent in DCT (f, arrow), and vacuolar degeneration is observed in PCT with severe dilation of lumen (f, arrowhead). ( $g, h)$ At 7 days after AL treatment, tubular regeneration is noted in TAL and DCT with dilation of lumen ( $g$ and $\mathrm{h}$, arrow). Bar $=50 \mu \mathrm{m}$. HE stain. 


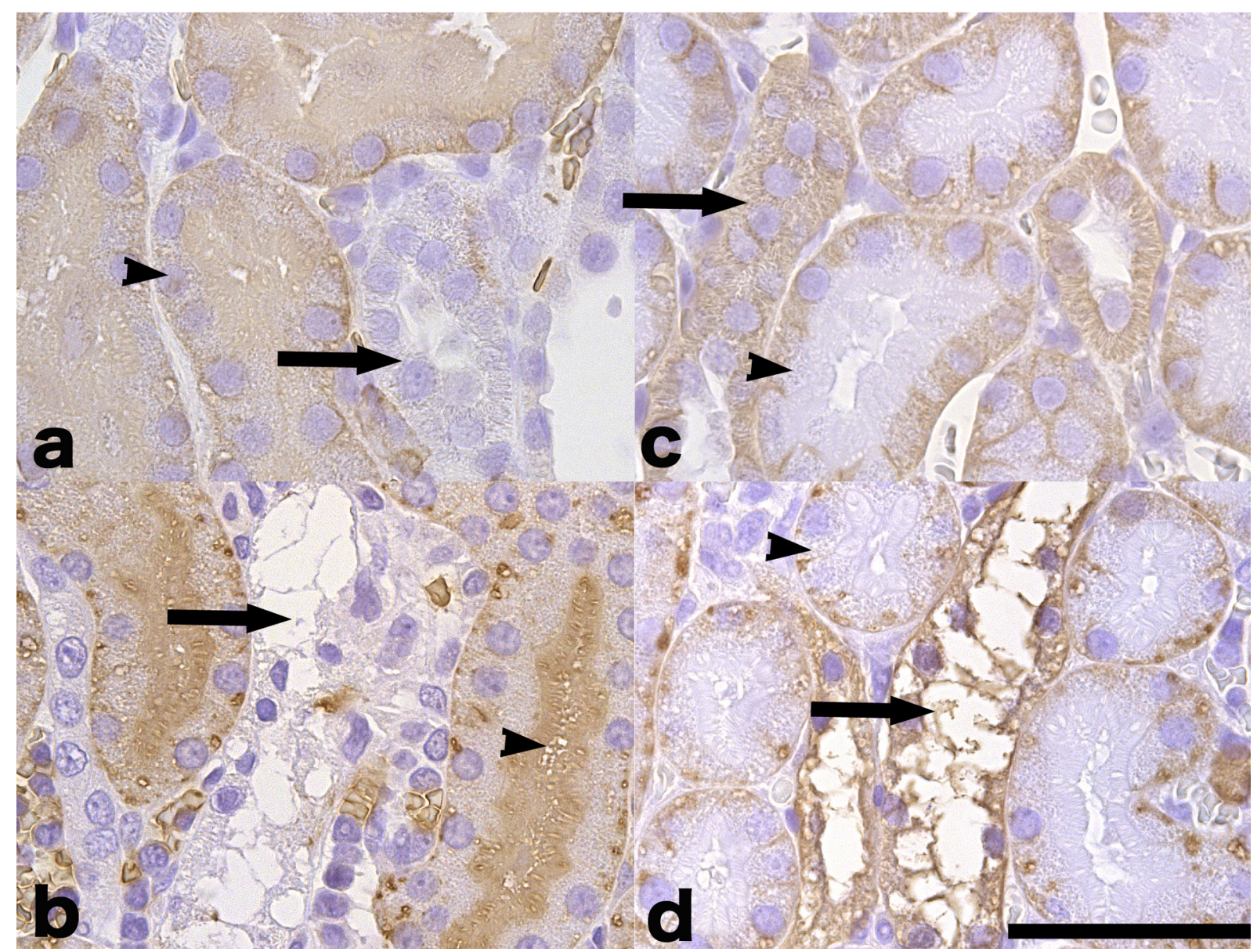

Fig. 2. AL initially causes degeneration of thick ascending limbs of Henle in the outer medulla. (a) AQP1 is localized in cytoplasm of the proximal tubule (arrowhead), but show a negative reaction in TAL (arrow) in the non-treated group. (b) At 24 hours after AL treatment, degenerated tubules correspond to AQP1-negative TAL (arrow), and the brush border of the proximal tubule shows strong AQP1 expression compared with cytoplasm (arrowhead). (c) Expression of $\mathrm{Na} / \mathrm{K}$ pump is localized on the basolateral of proximal tubule (arrowhead), but is distributed in all of the cytoplasm of TAL (arrow) in the non-treated group. (d) At 24 hours after AL treatment, degenerated tubules with strong $\mathrm{Na} / \mathrm{K}$ pump expression corresponds to TAL (arrow). The positivity level of the proximal tubule is decreased compared with that in the non-treated group (arrowhead). Bar $=50 \mu \mathrm{m}$. Immunohistochemical staining for AQP1 and Na/K pump and hematoxylin counterstain.

GLUT2 glucose transporter in the renal tubule is mainly located at the basolateral membrane of proximal tubular epithelial cells ${ }^{7}$. If AL induces tubular damage via GLUT2, only the proximal tubule may be damaged. However, in our study, the primarily affected tubule after AL treatment was the TAL. AL could interact and generate reactive oxygen species in a redox cycle in the plasma membrane. TAL is exquisitely sensitive to hydroxyl radicals and peroxide generation. Thus it is possible that they might show early evidence of degeneration, despite whether or not AL is transported. According to the expansion of the lesions, both the proximal tubule and distal tubule were affected. The precise mechanism of the expansion is unknown, but proximal tubule damage may be caused by AL entering tubular cells via the glucose transporter GULT2. The earliest pancreatic beta cell changes in AL-treated animals are localized to the mitochondria, which shows swelling and disruption of inner and occasionally outer membranes ${ }^{8}$. In our study, mitochondria and cytoplasm showed slight swelling in TAL that is the initial affected tubule at 8 hours after AL treatment, and severe swelling of cells was also observed in TAL at 24 hours after AL treatment. These results suggest that AL causes both mitochondrial and cellular membrane toxicity in TAL without involving GLUT2.

In conclusion, our present data suggest that $\mathrm{AL}$ initially causes degeneration of the thick ascending limbs of Henle, and induces mitochondrial and cellular damage in the tubular epithelium without involving GLUT2.

Disclosure of Potential Conflicts of Interest: The authors declare that they have no competing interests. 


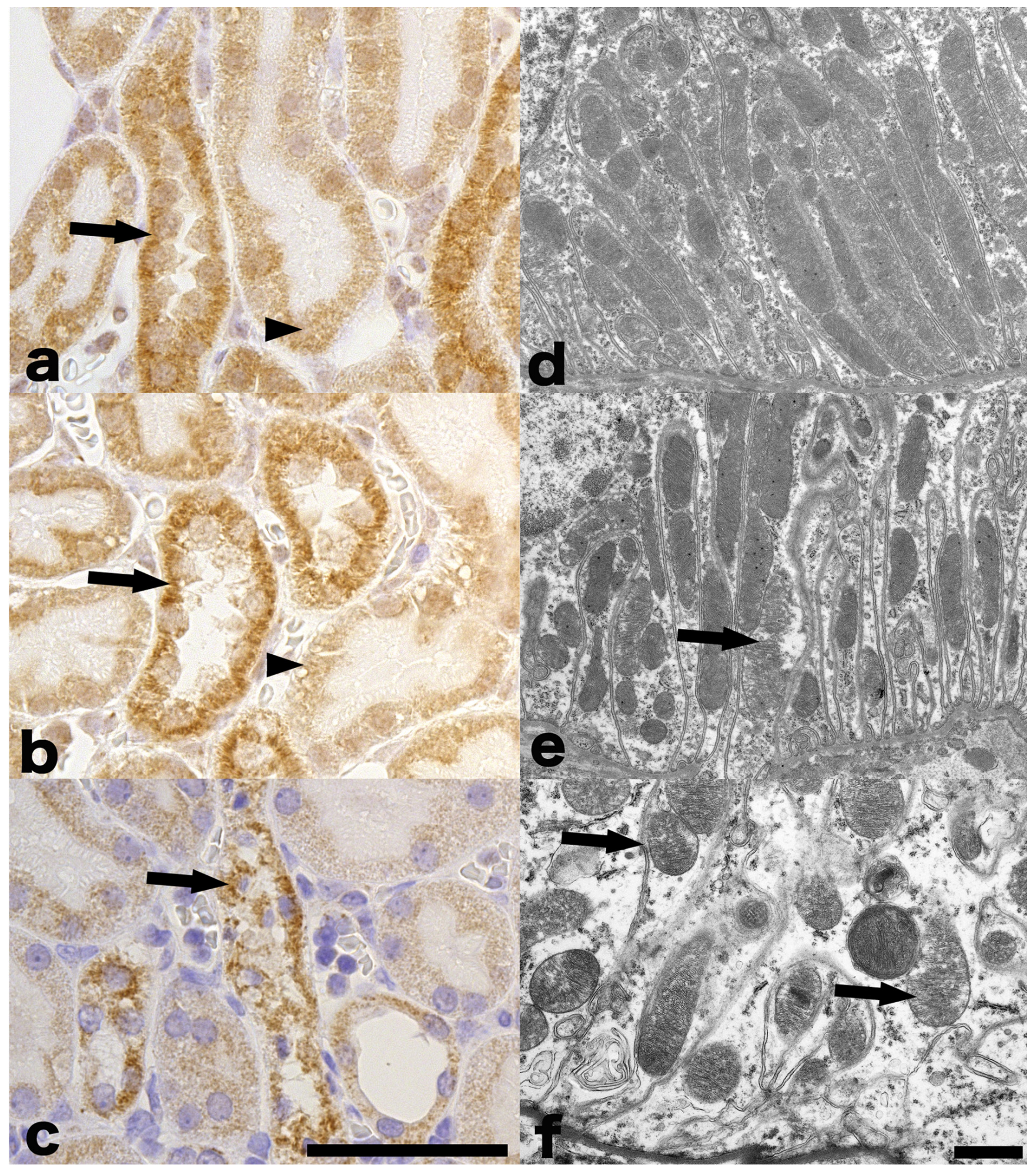

Fig. 3. AL induces mitochondrial and cellular damage in the tubular epithelium. (a) Expression of prohibitin in TAL (arrow) is stronger than that in the proximal tubule (arrowhead) in the non-treated group. (b) At 8 hours after AL treatment, positivity in TAL (arrow) and the proximal tubule (arrowhead) is the same as in the non-treated group. (c) At 24 hours after AL treatment, prohibitin-positive granules in TAL varied in size (arrow) and the staining intensity decreased compared with those in the non-treated group. Bar $=50 \mu \mathrm{m}$. Immunohistochemical staining for prohibitin and hematoxylin counterstain. (d) Extensive interdigitation with elongated mitochondria is observed in TAL in the non-treated group. (e) At 8 hours after AL treatment, the lesions of TAL are characterized by rarefaction of cytoplasm and slight swelling of mitochondria (arrow). (f) At 24 hours after AL treatment, severe swelling of cytoplasm and mitochondria (arrows) and irregular basal interdigitation are observed in TAL. Bar $=1 \mu \mathrm{m}$. Uranyl acetate and lead citrate. 


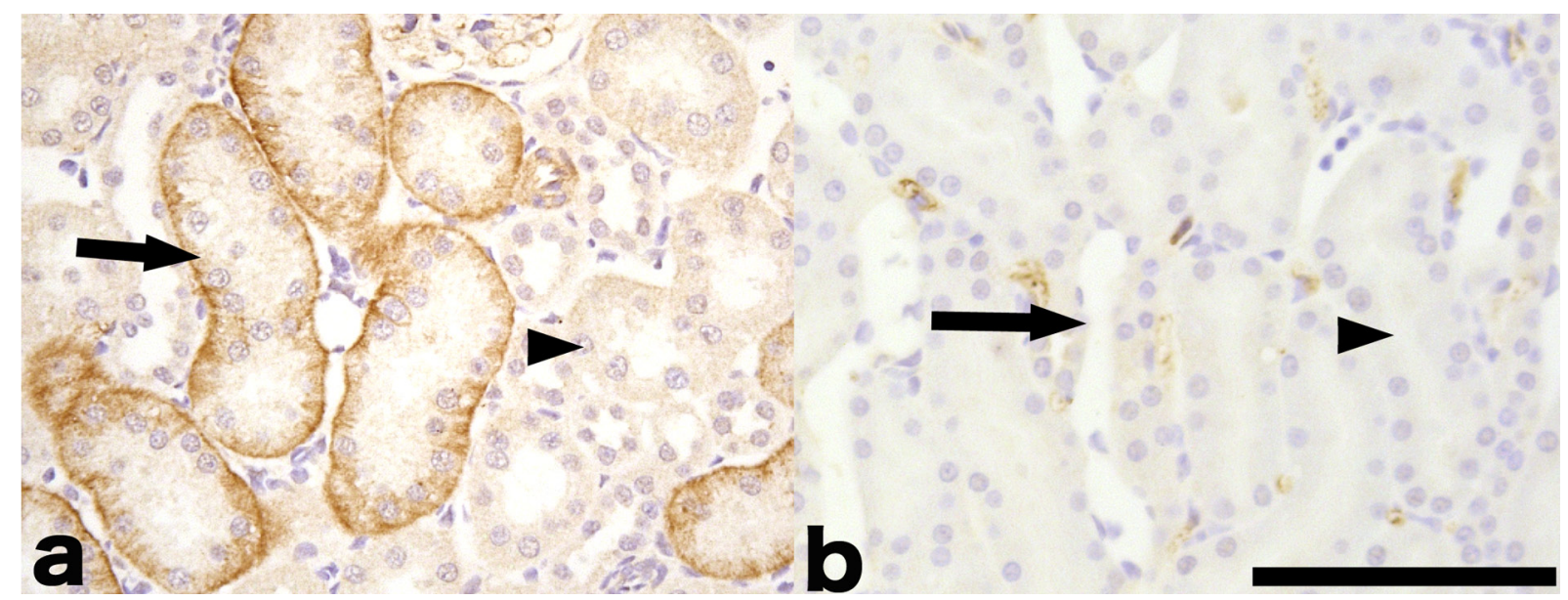

Fig. 4. AL induces damage in the tubular epithelium not via GLUT2. GLUT2 is expressed at the basolateral membrane of PCT in the cortex (a, arrow), but there is no expression in the pars recta ( $b$, arrowhead), TAL (b, arrow), and distal tubule in the outer medulla (a, arrowhead) in the non-treated group. Bar $=50 \mu \mathrm{m}$. Immunohistochemical staining for GLUT2 and hematoxylin counterstain.

\section{References}

1. Evan AP, Mong SA, Connors BA, Aronoff GR, and Luft FC. The effect of alloxan, and alloxan-induced diabetes on the kidney. Anat Rec. 208: 33-47. 1984. [Medline] [CrossRef]

2. Vargas L, Friederici HH, and Maibenco HC. Cortical sponge kidneys induced in rats by alloxan. Diabetes. 19: 33-44. 1970. [Medline] [CrossRef]

3. Bruckmann G, and Wertheimer E. Alloxan studies; the action of alloxan homologues and related compounds. J Biol Chem. 168: 241-256. 1947. [Medline]

4. Zhang L, Terayama Y, Nishimoto T, Kodama Y, and Ozaki $\mathrm{K}$. Acute alloxan toxicity causes granulomatous tubulointerstitial nephritis with severe mineralization. J Toxicol Pathol. 29: 261-264 2016. [CrossRef]

5. Beveridge JM, and Johnson SE. Studies on diabetic rats: the production of cardiovascular and renal disease in diabetic rats. Br J Exp Pathol. 31: 285-293. 1950. [Medline]

6. Bauchet AL, Masson R, Guffroy M, and Slaoui M. Immunohistochemical identification of kidney nephron segments in the dog, rat, mouse, and cynomolgus monkey. Toxicol Pathol. 39: 1115-1128. 2011. [Medline] [CrossRef]

7. Lenzen S. The mechanisms of alloxan- and streptozotocininduced diabetes. Diabetologia. 51: 216-226. 2008. [Med- line] [CrossRef]

8. Boquist L. The endocrine pancreas in early alloxan diabetes. Including study of the alloxan inhibitory effect of feeding and some hexoses. Acta Pathol Microbiol Scand [A]. 85A: 219-229. 1977. [Medline]

9. Boquist L, and Boström T. Alloxan effects on mitochondria in vitro, studied with regard to inhibition of mitochondrial aconitase. Diabete Metab. 11: 232-237. 1985. [Medline]

10. Bedford JJ, Leader JP, and Walker RJ. Aquaporin expression in normal human kidney and in renal disease. J Am Soc Nephrol. 14: 2581-2587. 2003. [Medline] [CrossRef]

11. Kashgarian M, Biemesderfer D, Caplan M, and Forbush B 3rd. Monoclonal antibody to Na,K-ATPase: immunocytochemical localization along nephron segments. Kidney Int. 28: 899-913. 1985. [Medline] [CrossRef]

12. Elsner M, Tiedge M, Guldbakke B, Munday R, and Lenzen S. Importance of the GLUT2 glucose transporter for pancreatic beta cell toxicity of alloxan. Diabetologia. 45: 1542-1549. 2002. [Medline] [CrossRef]

13. Weaver DC, McDaniel ML, and Lacy PE. Alloxan uptake by isolated rat islets of Langerhans. Endocrinology. 102: 1847-1855. 1978. [Medline] [CrossRef]

14. Gorus FK, Malaisse WJ, and Pipeleers DG. Selective uptake of alloxan by pancreatic B-cells. Biochem J. 208: 513515. 1982. [Medline] [CrossRef] 